INPLASY

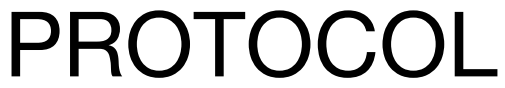

To cite: Li et al. Efficacy and safety of Shufeng Jiedu capsule for coronavirus disease 2019 (COVID-19): A protocol for systematic review and meta-analysis. Inplasy protocol 202070024. doi: 10.37766/inplasy2020.7.0024

Received: 08 July 2020

Published: 08 July 2020

Corresponding author: Runmin Li

Irmzyy@163.com

Author Affiliation:

Shandong University of

TraditionalChinese Medicine

Support: We have no financial support.

Review Stage at time of this submission: The review has not yet started.

Conflicts of interest:

The authors have no conflicts of interests to disclose.

\section{Efficacy and safety of Shufeng Jiedu capsule for coronavirus disease 2019 (COVID-19): A protocol for systematic review and meta-analysis}

Li, R' Li, Y2; Li, B³; Sun, H4; Liu, X5; Ge, X6; Liu, Y7; Yang, J8.

Review question / Objective: To evaluate the efficacy and safety of Shufeng Jiedu capsule for coronavirus disease 2019 (COVID-19).

Condition being studied: From the end of 2019 to the present, coronavirus disease 2019 (COVID-19) has put considerable pressure on the world's medical system and caused significant mortality and economic losses around the world. In China, the Shufeng Jiedu capsule has been widely used in the treatment of COVID-19, but there is still a lack of evidencebased medical evaluation.

Information sources: China National Knowledge Infrastructure (CNKI), the Wanfang database, the Chinese Scientific Journals Database (VIP), PubMed, Embase and the Cochrane Library will be searched for relevant literature, regardless of publication date or language.

INPLASY registration number: This protocol was registered with the International Platform of Registered Systematic Review and Meta-Analysis Protocols (INPLASY) on 08 July 2020 and was last updated on 08 July 2020 (registration number INPLASY202070024).

\section{INTRODUCTION}

Review question / Objective: To evaluate the efficacy and safety of Shufeng Jiedu capsule for coronavirus disease 2019 (COVID-19).
Condition being studied: From the end of 2019 to the present, coronavirus disease 2019 (COVID-19) has put considerable pressure on the world's medical system and caused significant mortality and economic losses around the world. In China, the Shufeng Jiedu capsule has been 
widely used in the treatment of COVID-19, but there is still a lack of evidence-based medical evaluation.

\section{METHODS}

Participant or population: Regardless of age or gender, patients with COVID-19 met the diagnostic criteria of the National Health and Health Commission of the People's Republic of China on the diagnosis and treatment of new coronavirus infection pneumonia (trial version 7). Specifically, confirmation of a case had to meet one of the following criteria: positivity for novel coronavirus nucleic acid by real-time fluorescence RTPCR; viral gene sequencing results that are highly homologous to the known novel coronavirus; and positivity for serum novel coronavirus-specific IgM and IgG antibodies.

Intervention: The treatment group was given Shufeng Jiedu capsule on the basis of conventional treatment. The control group was only given routine treatment. Routine treatment mainly includes antiviral therapy, such as oral lopinavir, ritonavir, remdesivir, chloroquine, hydroxychloroquine and arbidol.

Comparator: The treatment group was given Shufeng Jiedu capsule on the basis of conventional treatment. The control group was only given routine treatment. Routine treatment mainly includes antiviral therapy, such as oral lopinavir, ritonavir, remdesivir, chloroquine, hydroxychloroquine and arbidol.

Study designs to be included: RCTs, regardless of publication status and language.

Eligibility criteria: The PICOS principles were given full consideration to establish the inclusion and exclusion criteria of this systematic review. Regardless of age or gender, patients with COVID-19 met the diagnostic criteria of the National Health and Health Commission of the People's Republic of China on the diagnosis and treatment of new coronavirus infection pneumonia (trial version 7). Specifically, confirmation of a case had to meet one of the following criteria: positivity for novel coronavirus nucleic acid by real-time fluorescence RT-PCR; viral gene sequencing results that are highly homologous to the known novel coronavirus; and positivity for serum novel coronavirus-specific IgM and IgG antibodies. The treatment group was given Shufeng Jiedu capsule on the basis of conventional treatment. The control group was only given routine treatment. Routine treatment mainly includes antiviral therapy, such as oral lopinavir, ritonavir, remdesivir, chloroquine, hydroxychloroquine and arbidol. The primary outcomes included the disappearance time of fever, rale, cough, wheezing, pulmonary function test score and mortality rate. Additional outcomes included CPIS, ADL, hs-CRP, PCT, IFN- $\gamma$, IL-4, white blood cell count and incidence of adverse events.

Information sources: China National Knowledge Infrastructure (CNKI), the Wanfang database, the Chinese Scientific Journals Database (VIP), PubMed, Embase and the Cochrane Library will be searched for relevant literature, regardless of publication date or language.

Main outcome(s): The primary outcomes included the disappearance time of fever, rale, cough, wheezing, pulmonary function test score and mortality rate.

Additional outcome(s): Additional outcomes included CPIS, ADL, hs-CRP, PCT, IFN- $v$, IL-4, white blood cell count and incidence of adverse events.

Data management: EndNote $\mathrm{X} 9$ was used to manage the retrieved studies. The study selection was divided into two steps, which were completed by two researchers (Bingchen Li and Haiyang Sun). Preliminary screening involved eliminating repeated and unqualified studies by reading the title and abstract. Rescreening involved reading through the full text and selecting the studies according to the inclusion and exclusion criteria. According to the Cochrane Handbook for Systematic 
Reviews of Intervention, the two researchers (Xinyu Liu and Xin Ge) extracted the author, publication time, participant number, age, sex, intervention measures, course of disease/treatment and outcome indicators, filled in the data extraction table, and compared them with each other.

Quality assessment / Risk of bias analysis: Two researchers (Bingchen $\mathrm{Li}$ and $\mathrm{Xin} \mathrm{Ge}$ ) assessed the quality of the included RCTs independently by utilizing the Cochrane risk of bias assessment tool. As specified by Cochrane Handbook V.5.1.0, the following sources of bias were considered: random sequence generation, allocation concealment, participant blinding, outcome assessor blinding, incomplete outcome data, selective reporting, and other sources of bias. Each domain was rated as having a high, low or unclear risk of bias as appropriate. The two reviewers resolved any disagreements through discussion, and a third reviewer (Runmin Li) was involved if a consensus could not be reached.

Strategy of data synthesis: The metaanalysis was performed with Review Manager 5.3 and STATA 14.2 software. The outcomes were mainly represented by the mean difference (MD) or odds ratio (OR) with $95 \%$ confidence intervals, and a $\mathrm{P}$ value $<0.05$ was considered significant. The Cochrane Q-test and 12 statistics were used to assess heterogeneity. When $P<0.1$ or $12>50 \%$ indicated statistical heterogeneity, a random effects model was used to calculate the outcomes; otherwise, the fixed effect model was considered.

Subgroup analysis: If there was high heterogeneity in the studies, we performed subgroup analyses to explore the differences in age, sex, interventions, and course of disease/treatment.

Sensibility analysis: To ensure robustness of the combined results, sensitivity analyses were performed to assess the impact of studies with a high risk of bias. We compared the results to determine whether lower-quality studies should be excluded.
Country(ies) involved: China.

Keywords: COVID-19, Shufeng Jiedu capsule, protocol, systematic review, meta-analysis.

Contributions of each author:

Author 1 - Runmin Li - Writing - original draft.

Author 2 - Ying Li - Writing - original draft. Author 3 - Bingchen Li - Data curation. Author 4 - Haiyang Sun - Data curation. Author 5 - Xinyu Liu - Data curation. Author 6 - Xin Ge - Data curation. Author 7 - Yuanxiang Liu - Writing - review \& editing.

Author 8 - Jiguo Yang - Writing - review \& editing. 